\title{
Bottom-up and top-down manipulations for multi-order photonic crystallinity in a graphene-oxide colloid
}

\author{
Tian-Zi Shen ${ }^{1}$, Seung-Ho Hong ${ }^{1}$, Bomi Lee and Jang-Kun Song
}

The recent discovery of structural coloration in aqueous graphene-oxide (GO) dispersions has increased the applicability of carbon-based two-dimensional materials. However, the origin of the photonic band-gap is still poorly understood, and its practical manipulation is still in an early developmental stage. Here, we demonstrate full-color reflection with first- and secondorder Bragg reflections in a GO dispersion, and we use two fundamental approaches to manipulate GO photonic crystals, namely, bottom-up and top-down manipulation by controlling the Debye length and using shear or surface fields, respectively. Nanoscopic tailoring of the electrostatic effective thickness and macroscopic smoothing of the curvature of the GO sheet result in similar modifications of the quality and pitch of the photonic crystallinity. Direct observation of the GO particle alignments reveals excellent electrostatic layer-to-layer packing assembly and rather poor in-layer assembly. These results elucidate the mechanism that governs the nematic nature of GO (rather than its lamellar mesophase) and the origin of its photonic crystalline periodicity and provide new methodologies for exploiting these attractive features in actual applications.

NPG Asia Materials (2016) 8, e296; doi:10.1038/am.2016.110; published online 12 August 2016

\section{INTRODUCTION}

Graphene oxide (GO) flakes, that is, 1-nm-thick disk-like particles, can be well-dispersed in water to form a stable colloid with spontaneous nematic assembly and non-linear anisotropic rheology. ${ }^{1-3}$ Nematic GO dispersions subjected to illumination typically exhibit a collective reflection of a brownish color from many GO particles, owing to the intrinsic absorption band of GO. ${ }^{4}$ However, color reflection throughout the entire visible spectrum was recently obtained from photonic crystals in a well-prepared nematic GO dispersion. ${ }^{5}$ Structural coloration has also been demonstrated in other colloidal systems such as in the swollen lamellar mesophase of plate-like particles with or without a supporting polymer network or surfactants ${ }^{6-8}$ the columnar phase of regularly sized plate-like particles $^{8-10}$ and the steric packing assembly of silica or polymer spheres or cubes. ${ }^{11-13}$ Nonetheless, color reflection in the GO dispersion is quite unexpected, considering that GO particles are highly polydisperse, irregularly shaped, curved in solution, ${ }^{14,15}$ and two orders of magnitude thinner than the photonic band-gap. From a practical point of view, photonic crystals potentially allow the use of GO in various optical applications, as long as an easy method is provided for manipulation. ${ }^{16}$ Finding ways to manipulate the reflection color and to achieve large-scale uniformity are, therefore, highly desirable for practical advances.

\section{MATERIALS AND METHODS}

GO dispersions

We prepared high-quality GO dispersions that exhibited structural coloration with a high oxidation rate using the Hummers' method (see Supplementary
Figure $\mathrm{S} 1$ for details of the GO samples). ${ }^{17}$ During the sample preparation, cleaning processes were repeatedly performed (that is, a total of 15 times) to remove residual salts; this removal was achieved by centrifuging the GO sample and replacing the supernatant liquid with pure deionized distilled water. ${ }^{18,19}$ Atomic force microscopy revealed that most of the flakes became single-layered after the cleaning process. The degree of oxidation in the GO sample was determined via X-ray photoelectron spectroscopy, as shown in Supplementary Figure S1. Scanning electron microscopy (SEM) revealed that the GO had a mean size of $\sim 6.5 \mu \mathrm{m}$. As reported by He et al., ${ }^{4}$ typical GO dispersions exhibit a brownish collective reflection from the many GO particles; however, well-prepared GO dispersions exhibit structural color reflection. ${ }^{5}$ This difference was clearly observable, as shown in Supplementary Figure S2.

Cells with undulated morphology

Most of the GO cells were fabricated using flat glass substrates. GO particles tend to align parallel to the surface due to surface effects. For comparison, we prepared an uneven cell, which was fabricated by using a substrate with undulating morphology. This uneven substrate was prepared by imprinting a fine sandpaper (800 Grit/25 Mesh) using polydimethylsiloxane and was subsequently used to fabricate a 1-mm-thick GO cell (see Supplementary Figure S3).

The spectral reflectance of the cells was measured using an integrating sphere-type reflective spectrometer (CM-2500d, Konica Minolta Co., Tokyo, Japan), and the 'spectral component excluded' (SCE) mode was selected to eliminate reflections from glass surface.

Freeze-drying field emission SEM analysis

A vial with a large neck containing a $0.4 \mathrm{wt} \% \mathrm{GO}$ dispersion was gently shaken to induce a circulating flow inside the vial to induce the uniform shear-alignment of the GO, parallel to the vial surface. The lid was removed, and the vial was partially submerged into liquid nitrogen, quickly freezing the

School of Electronics and Electrical Engineering, Sungkyunkwan University, Gyeonggi-do, Korea

1 These authors contributed equally to this work.

Correspondence: Professor J-K Song, School of Electronics and Electrical Engineering, Sungkyunkwan University, 2066 Seoburo, Jangan-gu, Suwon, Gyeonggi-do 440-746, Korea.

E-mail: jk.song@skku.edu

Received 11 February 2016; revised 7 June 2016; accepted 12 June 2016 
aqueous GO dispersion. Then, the vial was kept in a freeze-drying vacuum chamber (UT-1000, EYELA Co., Tokyo, Japan) for $>12 \mathrm{~h}$ until the ice was perfectly sublimated. During the sublimation, several cracks appeared in the dried GO monolith, and the fractured GO monoliths were carefully removed from the vial using tweezers to prevent any compressional force on the samples.

The fractured monoliths were loaded on a metal mount with carbon tape and were coated with Pd by sputtering. Then, the samples were analyzed using a field emission SEM (JSM 7000F, JEOL Ltd., Tokyo, Japan).

\section{Confocal laser scanning microscopy}

Individual GO particles with a mean size of $6.5 \mu \mathrm{m}$ were poorly resolved when analyzed using confocal laser scanning microscopy (CLSM). Therefore, we prepared another aqueous GO dispersion using large-sized graphite powder. The large GO particles were discernible depending on the concentration of GO. The mean diameter was $\sim 27 \mu \mathrm{m}$; however, the sample was highly polydisperse, and many particles were larger than $100 \mu \mathrm{m}$.

An erected-type CLSM (405-nm laser; K1-Fluo, Nanoscope Systems Company, Daejeon, Korea) was used, and the pin-hole was set to 0.5 a.u. The objective had a magnification of $\times 40$ and a numerical aperture of 1.2.

\section{RESULTS}

First- and second-order Bragg reflections

A weak deep-blue-colored reflection appeared at a concentration of $1.3 \mathrm{wt} \%$ when water was slowly added into a 4 -wt $\%$ dark GO dispersion and was mixed evenly with a stirrer bar. Moreover, a series of iridescent colored reflections was obtained in the GO samples (as shown in Figure 1a) with the further addition of water. A different set of iridescent colored reflections, corresponding to the second-order Bragg reflection band, occurred in the low-concentration range, that is, $c a .0 .4-0.11 \mathrm{wt} \%$. These reflections were indicative of the high-quality periodicity associated with the GO dispersions, even at low concentrations. The pitches of the GO photonic crystals that yielded first- and second-order Bragg reflections were in the ranges of $150-260 \mathrm{~nm}$ and $300-520 \mathrm{~nm}$, respectively, at optical wavelengths of $400-700 \mathrm{~nm}$.

After 4 days of storage at room temperature, the color corresponding to the first-order reflection persisted, albeit with a slight fading of the color purity, whereas the second-order reflection was no longer observed (Figure $1 \mathrm{~b}$ ). The $\mathrm{C}-\mathrm{C}$ bond in the GO particles is spontaneously cleaved in aqueous solutions and releases $\mathrm{H}^{+}$ions that enhance the electrostatic screening effect and lead to reductions in the Debye length. ${ }^{1,19}$ Therefore, with increasing ion concentration, the second-order Bragg reflection (which has a longer periodicity) deteriorated before its first-order counterpart. As such, the second-order color reflection was recovered, and the color purity of the first-order samples improved (Figure 1c) when the ions were removed; this removal was achieved by centrifuging the degraded samples and replacing the supernatant water with pure distilled water. ${ }^{19,20}$ This indicates that the electrostatic repulsion between GO particles, owing to their electric double layer (EDL), extends $>0.5 \mu \mathrm{m}$ in the natural sample, which is free of polymer additives and consists of unmodified functional groups.

The corresponding reflection spectra are shown in Figure 1d and e; the reflection peak blue-shifted with increasing concentration. Figure 1f shows the peak wavelength $\left(\lambda_{\mathrm{P}}\right)$ as a function of the reciprocal volume fraction $(1 / \Phi)$; it shows a linear correlation between $\lambda_{\mathrm{P}}$ and $1 / \Phi$ in each section of the first- and second-order Bragg reflection regions. The interlayer spacing $(d)$ can be calculated by $\left(m \times \lambda_{\mathrm{P}} / n\right)$, where $m$ is the order of the Bragg reflection and $n$ is the refractive index of water. The interlayer spacing, $d$, is linearly proportional to $1 / \Phi$, as shown in Figure 1g. The slope was $\sim 0.8$, which roughly corresponds to the thickness of the GO particles.

a

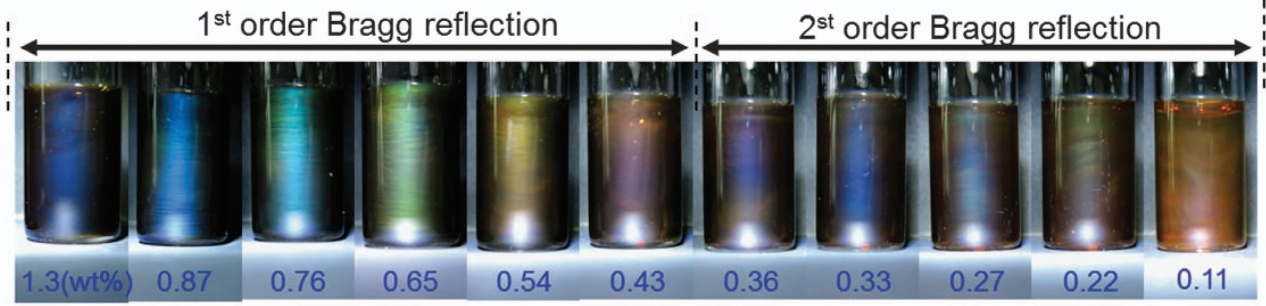

b

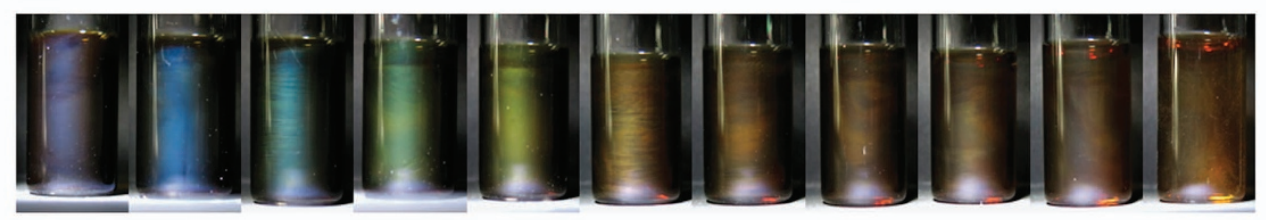

d

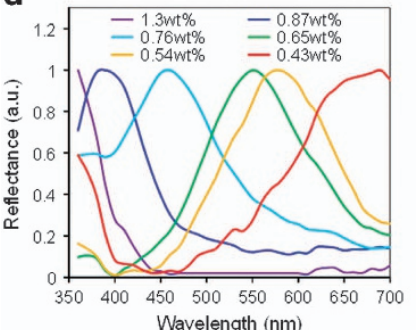

e

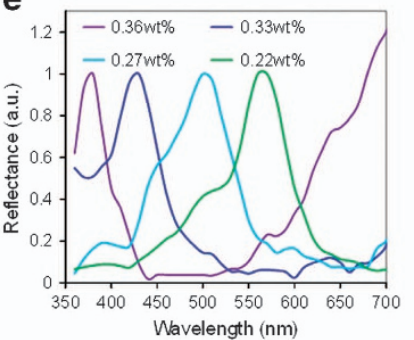

f

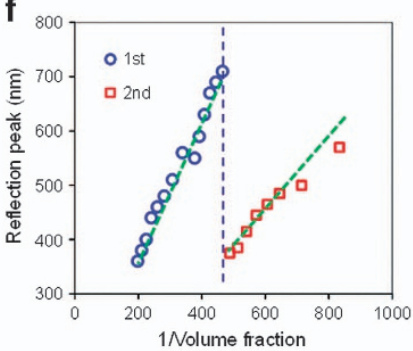

C
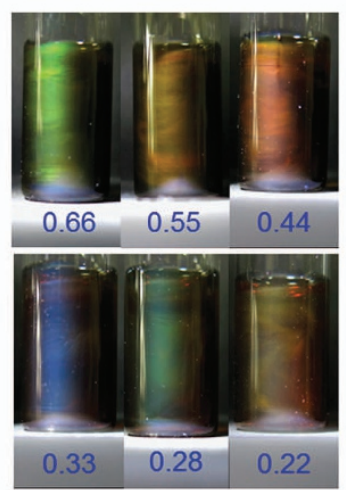

Figure 1 First- and second-order Bragg reflections in GO dispersions. (a) Well-prepared GO dispersions exhibited first- and second-order structural colorations. (b, c) The colors were partially faded after 4 days of storage (b) but were recovered after cleaning (c). (d, e) Normalized reflection spectra for the first- (d) and the second- (e) order Bragg reflections. (f) Peak wavelengths of reflections as a function of the reciprocal volume fraction. (g) Interlayer spacing estimated from the spectra as a function of the reciprocal volume fraction. 
Bottom-up control of the Debye length using ion addition and reduction

We performed several experiments to clarify the EDL effect on the photonic crystal structure. First, we added a small amount of $\mathrm{NaCl}$ to the GO dispersion. The reflection spectrum broadened, and the spectral peak blue-shifted (Figure 2a) with increasing ionic concentration; however, the structural color was destroyed with further additions. The increase in the ionic density enhances the electric screening effect in the colloid, thereby resulting in a decrease in the Debye length. This was verified by zeta potential analysis, the absolute value of which decreased with increasing ionic density (Supplementary Figure S4). The addition of $\mathrm{NaOH}$ resulted in relatively weak changes; $\mathrm{H}^{+}$ions were neutralized by $\mathrm{OH}^{-}$with small additions of $\mathrm{NaOH}$.

Spectral broadening and blue-shifting also occurred when the GO cells were exposed to ultraviolet (UV) irradiation (Figure 2b). It is known that UV radiation reduces GO, and the reduced GO is electrically conductive. ${ }^{21,22}$ Reduction indicates a decrease in the functional group density on the GO surface, which was confirmed by X-ray photoelectron spectroscopy analysis (see Supplementary Figure S5). The decrease in the functional group density was accompanied by a decrease in the surface charge density, which was also confirmed by the decreasing absolute value of the zeta potential with an increasing duration of UV irradiation (Supplementary Figure S4). The decrease in the surface charge density weakens the electrostatic repulsion and leads to a decrease in the Debye length. Therefore, a reduction in the periodicity of the lamellar structure is induced by either reducing the surface charge density or increasing the ionic concentration. The reduction of the Debye length expels water from the interparticle space, and the expelled water then forms voids filled with water, as shown in Figure $2 \mathrm{c}$. The size of the voids depends on the initial GO concentration; the CLSM image and the inset image in Figure $2 \mathrm{c}$ were acquired from 0.2 and $0.02 \mathrm{wt} \% \mathrm{GO}$ dispersions after 5 days' storage.

\section{Shear field induced uniform color reflection}

The structural colors in the vials were non-uniform and exhibited irregularly undulating swirling patterns that corresponded to the shear-induced flow-mark. ${ }^{23}$ When the GO dispersion was intentionally stirred with a glass rod, the flow trajectory remained on the surface (Figure 3a). The purity of the structural color adjacent to the flow-mark was higher than that of other regions in the vial, indicating that the shear-induced GO alignment improved the quality of the structural periodicity. The generation of flow-marks during the preparation of a fluidic colloid is unavoidable.

Top-down manipulations of GO photonic crystals can be achieved via a shear field. The vials containing GO dispersions were slowly rolled as a simple means of determining the effect of the shear field on the alignment of GO. This slow and gentle rolling induces a shear field near the surface of the vial, thereby resulting in a uniform structural color reflection of considerable color purity (Figure $3 \mathrm{~b}$ ). The figure shows that both the macroscopic flow-mark pattern and the quality of the nanoscale periodicity were influenced by the shear fields.

We performed a systematic investigation of the shear effect using simple GO dispersion cells that were fabricated in-house. During the fabrication, a GO dispersion droplet was dropped on a bare glass substrate with a 1-mm-thick rectangular polydimethylsiloxane wall, and this substrate was then covered with another glass substrate. The cells exhibited structural colors but had, as expected, an irregular flowmark (top row of the images in Figure 3c). A shear force was then imposed by sliding the upper glass substrate from one edge to the other, as illustrated in Figure $3 \mathrm{~d}$. This resulted in the improved uniformity of the reflected color in the entire area (bottom row of the images in Figure 3c).

The spectral reflectance underwent two modifications owing to the action of the shear force (Figure 3d). First, the full width at half maximum was reduced by $27 \%$ in the red region $(140-102 \mathrm{~nm})$, by $11 \%$ in the green region $(149-133 \mathrm{~nm})$ and by $0 \%$ in the blue region $(90 \mathrm{~nm})$. This reduction was expected because the shear stress may smooth out the wrinkles in the macroscopic GO alignment. Compared with the structural reflection from a smooth alignment, the reflection from an undulated alignment is expected to consist of light from a wider range of wavelengths. Therefore, the ironing of wrinkles will lead to a reduction in the full width at half maximum. This reduction was more apparent in longer pitch structures than in shorter ones, indicating that the periodic lamellar structure was softened with a decreasing GO concentration. Second, the blue shift in the spectral peak constitutes a contrasting effect to that of the flattened GO a
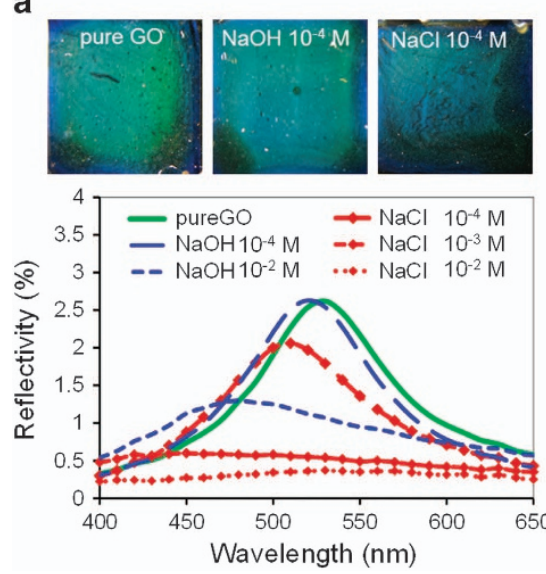

b

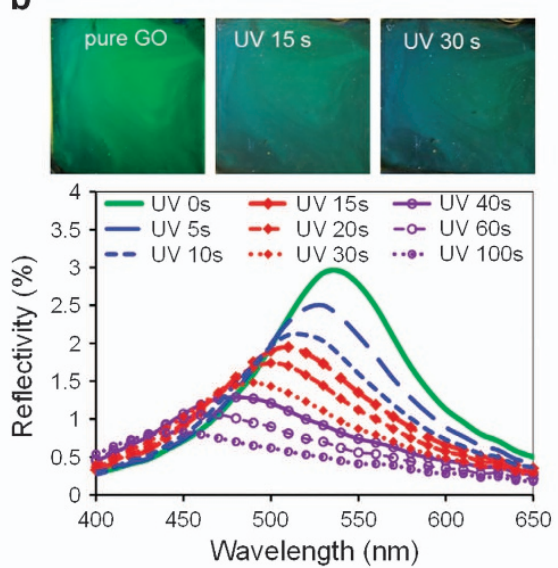

c

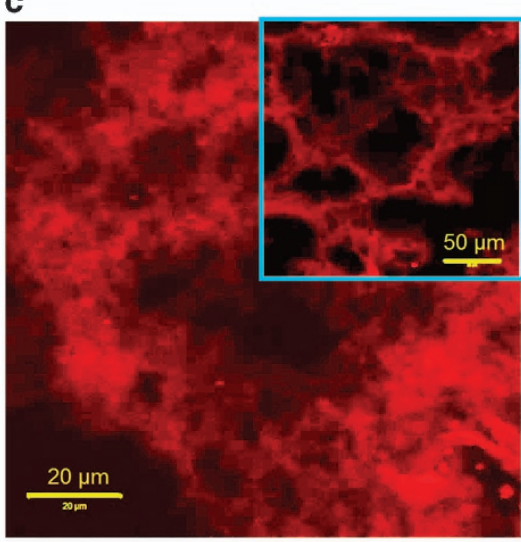

Figure 2 Bottom-up manipulation of structural color by ionic addition and UV exposure. (a) The peak in the reflection underwent a blue-shift, and its intensity decreased with increasing ionic concentration. (b) A similar color shift and degradation were observed when the sample was exposed to UV light. Each of the samples shown in the photos has an area of $2.0 \times 2.0 \mathrm{~cm}^{2}$. (c) After 5 days' storage of the 0.2 wt\% GO dispersion, voids appeared with the disappearance of structural color reflection. The voids were composed of the water expelled from the interparticle space. The sizes of the voids were larger in low-concentration (0.02 wt\%) GO dispersions (inset). 


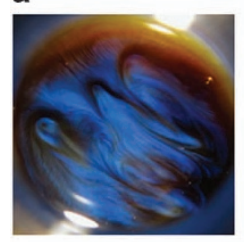

b

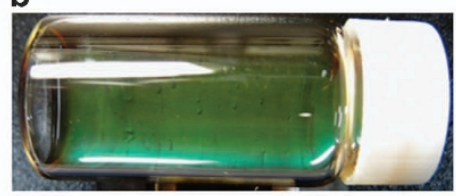

e
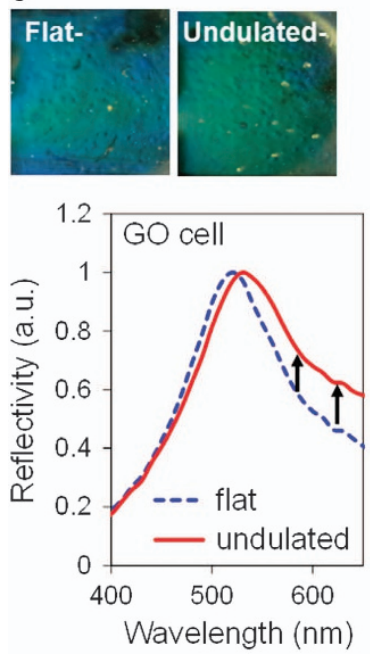

f

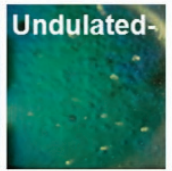

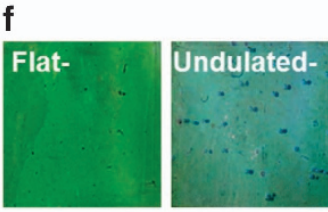

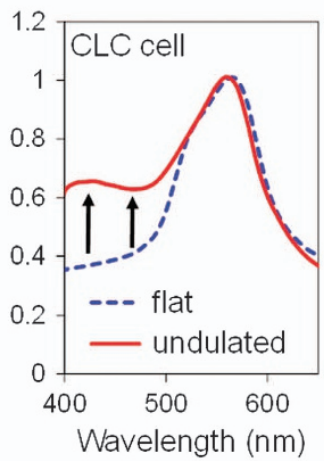

C

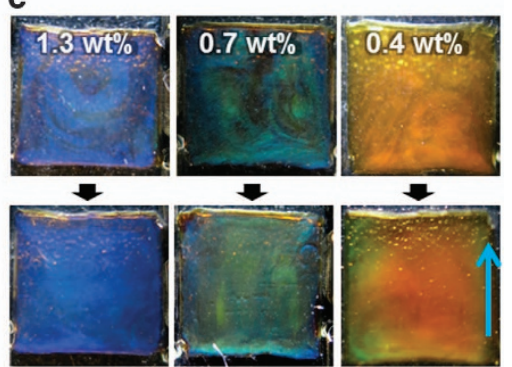

d

Gentle slide

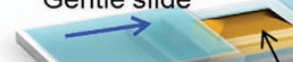

GO dispersion

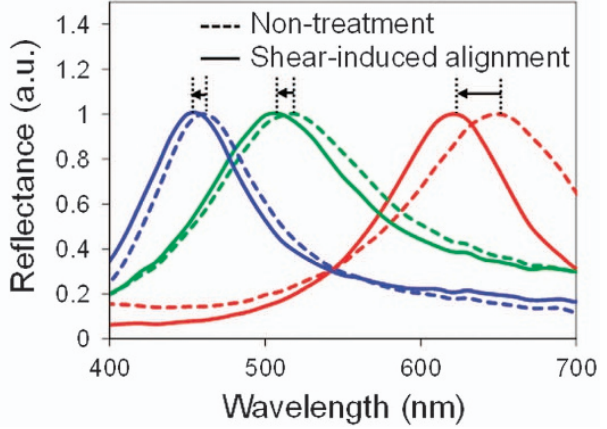

Figure 3 Top-down manipulation of structural color using shear and surface fields. (a) The structural color was influenced by the flow effect. The flow-mark was created using a stick. (b) Uniform and pure color reflections were obtained by rolling the vials. (c, d) The uniformity and color-purity of the structural color reflection were improved by gentle sliding of the cover glass (cell size: $2 \times 2 \mathrm{~cm}^{2}$ ). (e) The spectrum changed before and after application of the shear field. The structural color from the GO dispersion became reddish after coming into contact with the uneven surface. (f) The color from the cholesteric liquid crystal (CLC) cell became bluish with the same surface treatment. The contrast reveals surface-induced smoothing of GO particles and the decrease in the periodicity.

alignment. Theoretically, the flattened photonic crystal structure is expected to result in a red shift, rather than in a blue shift (see 'Surface field effect' in the next section). We thus conclude that the blue shift arises from a reduction in the structural periodicity of the GO alignment rather than from the flattening of the long-range alignment modulation. Spectral shifts of 29, 12 and $9 \mathrm{~nm}$ for the red, green and blue regions correspond to reductions of $11,4.6$ and $3.4 \mathrm{~nm}$, respectively, in the periodicity. This verifies the softening of GO packing at low concentrations.

\section{Surface field effect}

The surface field provides another method for top-down manipulation. CLSM revealed that the GO particles near the surface were aligned approximately parallel to the surface (not shown here). However, those in the middle of the cell were aligned in an undulating manner, and some were even vertical with respect to the surface. To clarify the surface effect, we prepared a highly uneven undulated substrate and fabricated a cell using this substrate (see Supplementary Figure S3). The uneven GO cell exhibited greater reflection over longer wavelengths, compared with its counterpart that had flat surfaces. Consequently, the bluish color in the flat cell became greenish in the undulated cell (Figure 3e). When the same uneven cell was filled with cholesteric liquid crystals (CLCs), however, the opposite color shift occurred (Figure 3f); the green color in the flat CLC cell became bluish in the uneven CLC cell. The photonic band gap in CLC arises from the molecular chiral helix, whose periodicity is not influenced by the surface morphology. The undulation-induced tilting of the helical axes, however, leads to the blue shift. ${ }^{24}$ In contrast, the periodicity of the lamellar alignment of the large GO sheets increased, owing to the effect of the undulating surface morphology.

\section{Effective GO thickness including the Debye length}

Excluded-volume theories based on steric interactions successfully elucidate the formation of the lyotropic mesophase and propose that the critical volume fraction for the phase transition $\left(\Phi_{\mathrm{T}}\right)$ is proportional to $T / D,{ }^{25,26}$ where $T$ and $D$ are the mean thickness and diameter, respectively, of the particles. ${ }^{3,26,27}$ In a colloid, the EDL acts as a large shell surrounding the bare particle, thereby resulting in a significant (that is, $>50$-fold) increase in the effective thickness of the particles. ${ }^{28}$ However, most studies have overlooked the EDL in Onsager-type calculations for GO liquid crystals, ${ }^{29-32}$ and $\Phi_{\mathrm{T}}$ was simply estimated by determining the aspect ratio of the dried GO sheet. By assuming that the effective thickness $\left(T_{\text {eff }}\right)$ is $m$ times that of the dried GO $\left(T_{\mathrm{GO}}\right)$ and using the approximation $\Phi_{\mathrm{T}} \sim 4.5 T / D$, the actual phase-transition concentration to the nematic phase including $\mathrm{EDL}\left(\Phi_{\mathrm{EDL}}\right)$ is given as:

$$
\Phi_{\mathrm{EDL}} \approx 4.5 \frac{T_{\mathrm{EDL}}}{D}=4.5 \frac{m T_{\mathrm{GO}}}{D}=m \Phi_{\mathrm{T}}
$$

Therefore, $\Phi_{\mathrm{EDL}}$ is $m$ times larger than $\Phi_{\mathrm{T}}$, which is calculated without taking the EDL into consideration. The corresponding GO concentration $\left(\Phi_{\mathrm{GO}}\right)$, excluding $\mathrm{EDL}$, is $\Phi_{\mathrm{EDL}} / m$, which is the same as $\Phi_{\mathrm{T}}$, according to equation (1). In other words, $\Phi_{\mathrm{T}}$ is not influenced by the inclusion of EDL in spite of the significant increase in the effective thickness. A more accurate calculation, which considers the polydispersity of GO, ${ }^{26}$ yields only a slight increase in $\Phi_{\mathrm{T}}$ by including 
the EDL. This inclusion leads to a decrease in the aspect ratio $\left(D / T_{\text {eff }}\right)$ but to an increase in the effective volume fraction. These two factors have contrasting impacts on the $\Phi_{\mathrm{T}}$, thereby resulting in only slight changes in $\Phi_{\mathrm{T}}$.

\section{High effective packing ratio and excellent layer-to-layer stacking assembly}

On the other hand, the increased volume packing ratio, owing to the inclusion of EDL, has a significant effect on the photonic crystallinity. Positional ordering in hard-particle colloids is typically attributed to the packing effect. ${ }^{12,33,34}$ After the prolonged centrifugation of the GO dispersion at 2200 r.p.m. to eliminate water, a 2-vol\% GO gel was obtained. In other words, centrifugation was ineffective in expelling the 98 -vol\% of water from the dispersion. By assuming that the remaining 98-vol\% water belongs to the rather hard core shell within the slippery boundary, it is estimated to be $\sim 25 \mathrm{~nm}$ from the GO surface. A similar value can be found by directly calculating the Debye thickness using the ionic concentration. ${ }^{19}$ The slippery boundary may be considered from the viewpoint of the packing ratio. For example, an effective packing ratio of $30 \mathrm{vol} \%$ is assumed for a $1.2-\mathrm{wt} \%(\sim 0.6 \mathrm{vol} \%) \mathrm{GO}$ dispersion that exhibits a pure blue reflection. This value is similar to the packing ratio at which periodic crystallinity can be induced. ${ }^{12,33,34}$ In the case of an excellent GO sample, the interparticle osmotic repulsion extends by up to $500 \mathrm{~nm}$ beyond the boundary, as confirmed in Figure 1a. If the Debye length beyond the slippery boundary is taken into consideration, then the ensuing increase in the ratio gives rise to excellent layer-to-layer periodicity. Unlike the pure steric interaction with on/off-type repulsion, the EDL-induced electrostatic repulsion is elastic, thereby allowing the continuously adjustable reflection color that varies with the GO concentration.

We prepared a $200-\mu$ m-thick GO cell with highly uniform GO alignment, which was achieved by combining the surface and shear fields. The color reflected by the cell shifted to blue with an increasing viewing angle (Figure 4) when a fixed white illumination is applied obliquely at $-30^{\circ}$ with respect to the sample normal. This result concurs (to some extent) with the result obtained from the simulation when a perfect one-dimensional photonic crystal, whose axis is normal to the surface, is assumed. Amorphous photonic crystals exhibit a rather weak dependency on the viewing angle. ${ }^{33}$ Hence, Figure 4 indicates that the layer-to-layer periodicity is surprisingly good.

Freeze-drying field emission scanning electron microscopic analysis for GO alignment

Drying the GO dispersion at room temperature caused a dramatic change in its structure; the volume shrank, with a loss of structural

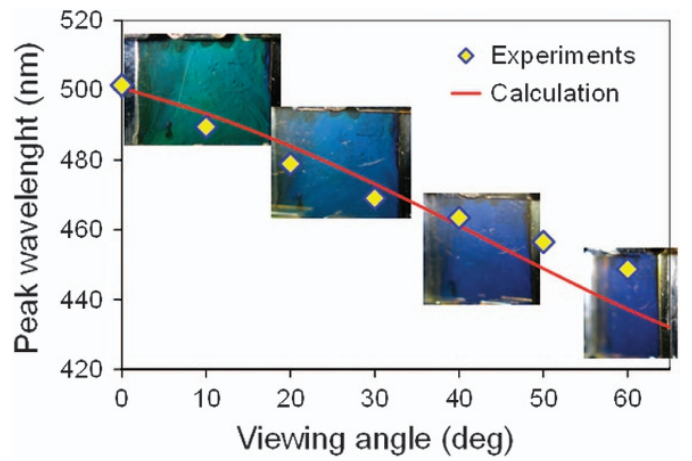

Figure 4 Dependence of structural color on the viewing angle. The structural color shifted to blue with an increasing viewing angle (cell size: $2 \times 2 \mathrm{~cm}^{2}$ ). color, and the GO particles adhered to each other to form packed wrinkly films. ${ }^{32,35}$ Figure 5 a shows the cross-sectional SEM image of a dried GO film with a wrinkle, which was made by drying a $1 \mathrm{wt} \% \mathrm{GO}$ droplet. The inset image shows closely packed GO sheets. The change is naturally expected because the electro-static repulsion between particles is expected to disappear with the recession and evaporation of water.

Quick freezing also could not prevent the alteration of the GO alignment. The $0.6 \mathrm{wt} \% \mathrm{GO}$ dispersion was analyzed using the freeze-drying fracture field emission scanning electron microscopic method. The GO dispersion with uniform shear-induced GO alignment displayed a clear green-colored reflection before freezing (Figure 5b). After the freeze-drying process, the dried GO monolith completely lost the initial structural color reflection and turned to a brown color (Figure 5c). SEM images of the sample surface in contact with the vial surface show structures different from those of the crosssectional view on the fractured surface, as shown in Figure 5d. Only the GO particles in contact with the vial surface were aligned parallel to the surface, as clearly indicated in Figure 5e. However, the SEM images of the side view, which were taken from the vertically cracked surface, exhibited very porous structures (Figure $5 \mathrm{f}-\mathrm{h}$ ). The porous structures were almost perfectly random in some areas (Figure 5f), and they exhibited elongation with periodic lines in other areas (Figure $5 \mathrm{~g}$ and $h$ ). The directions of elongation were not parallel to the vial surface, but were vertical, with tilting and bending (see the white dashed lines in Figure $5 \mathrm{~g}$ and $\mathrm{h}$ ).

The porous cork-like structure was distinct from the lamellar structure with excellent periodicity. Even the elongated structure was not parallel to the vial surface, and the pitch was $\sim 10 \mu \mathrm{m}$, which was in complete contrast with the periodic structures for the photonic crystals with structural color reflection. It has been reported that the freezing of water changes the morphological properties of a GO assembly and that the growth of ice is responsible for the elongated network structures. ${ }^{36,37}$ Hence, the freeze-dried GO film does not retain the initial GO alignment within the aqueous dispersion before drying. ${ }^{5,38,39}$ Removal of the water between the GO particles, via either evaporation or sublimation, significantly modifies the interparticle interactions and the GO alignment structure.

\section{CLSM analysis for the curvature of individual GO particles}

CLSM analysis can overcome the aforementioned issue and enable the direct observation of the GO alignment within the wet dispersion (Figure 6). The cell containing the $0.25 \mathrm{wt} \%$ GO dispersion (Figure 6a) showed a largely undulating GO alignment, and in particular, the side view of the vertically aligned GO particles explicitly revealed the particle curvatures in the GO liquid crystal. Some of the particles were almost flat (expanded inset (i)), whereas others were significantly curved (expanded inset (ii)). ${ }^{14}$ It is apparent that the space between the flat particles is narrower and more uniform than that between the curved particles. The inset image (iii) shows the nematic alignment of irregular GO particles; in which, the edge lines of neighboring GO particles do not match, and the GO particles are stacked randomly. Shear treatment caused the particles to align parallel to the substrate, as indicated in Figure 6b. Closely packed GO sheets appear brighter than the sparsely packed sheets or the sheets with missing layers because of the characteristics of the CLSM method. In the $0.45 \mathrm{wt} \% \mathrm{GO}$ dispersion, individual particles were faintly discernible (Figure $6 \mathrm{c}$ and $\mathrm{d}$ ). The untreated cell had an undulating GO alignment (Figure 6c), and the shear-treated cell had an improved GO alignment parallel to the substrates, which can be inferred from the uniform brightness profile (Figure $6 \mathrm{~d}$ ). For the higher- 

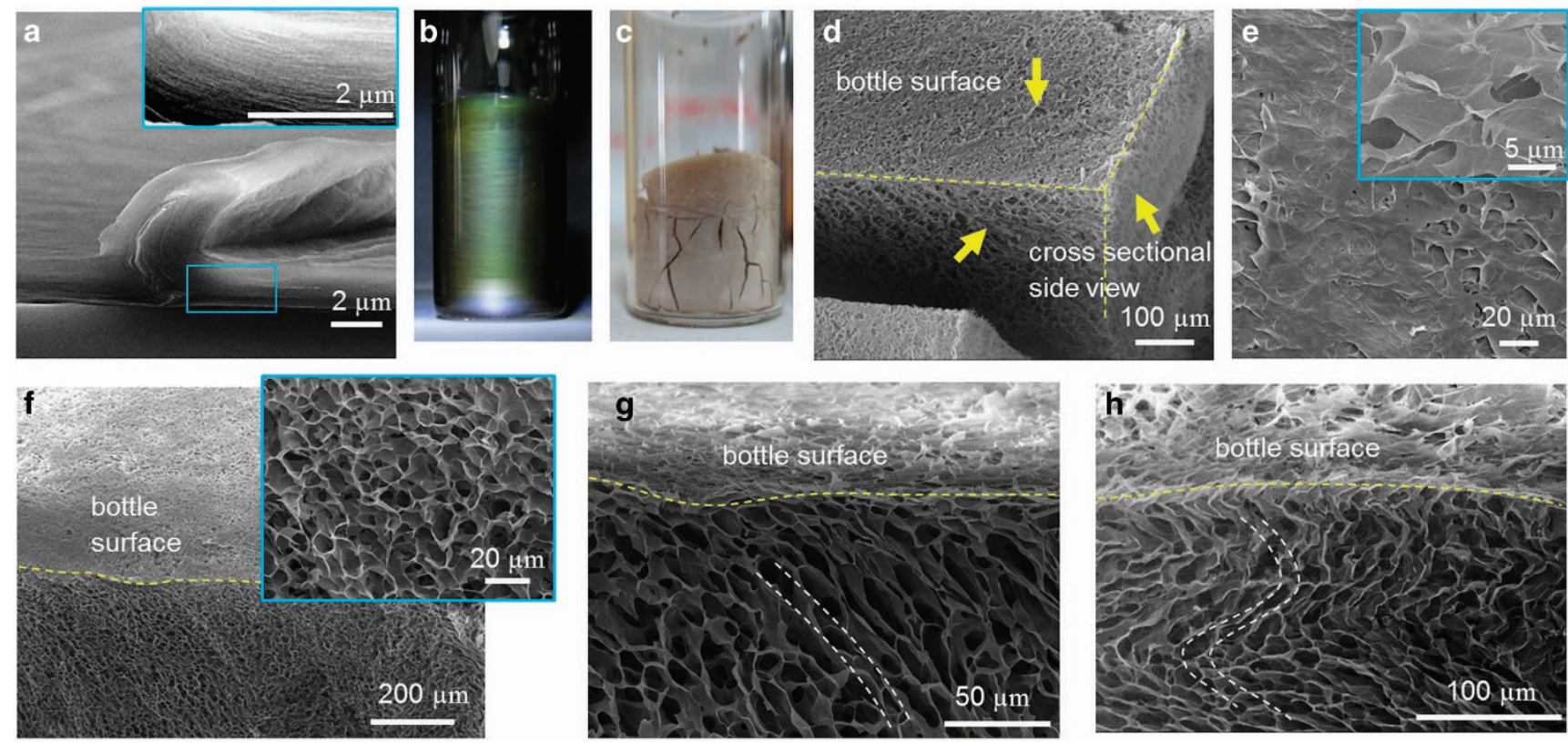

Figure 5 Field emission scanning electron microscopic analyses of ambient dried and freeze-dried GO samples. (a) A 1 wt\% GO droplet was dried at ambient conditions, and the fractured, dried film was analyzed. The cross-sectional diagram exhibits densely packed GO sheets. (b, c) 0.4 wt $\%$ GO dispersions before and after freeze-drying, respectively. (d-h) Fractured, freeze-dried GO monoliths were analyzed. The top surface, which was in contact with the vial surface, shows GO assembly parallel to the surface (e) but the fractured cross-sections have porous cork-like structures: random porous (f), obliquely elongated (g) or bent elongated structures (h). None of these exhibited periodic photonic crystal structures.

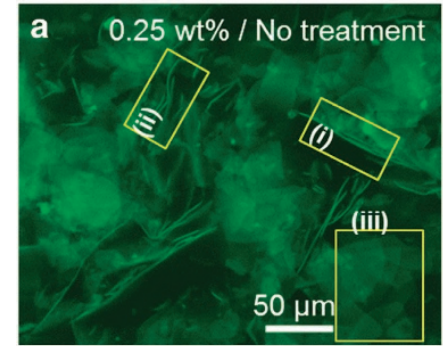

b $\quad 0.25 \mathrm{wt} \% /$ shear-treated

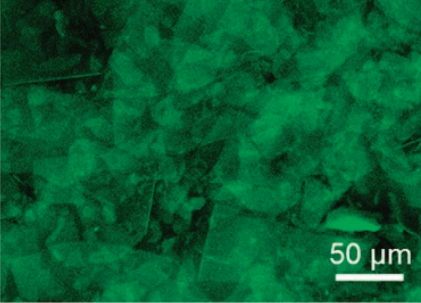

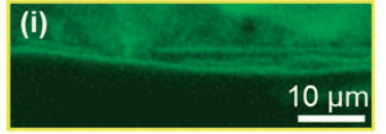
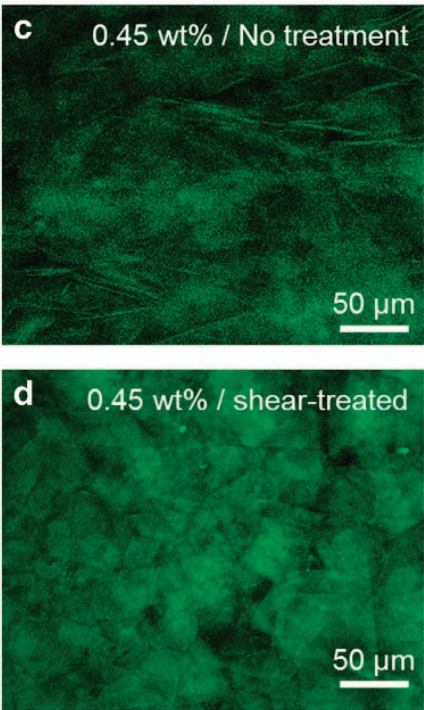

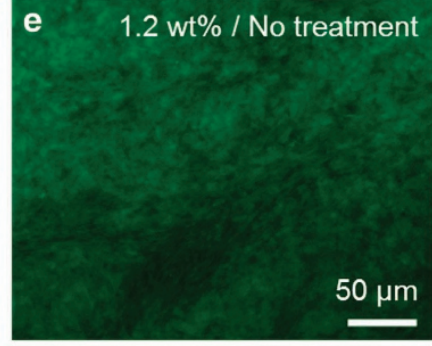

f $\quad 1.2 w t \% /$ shear-treated

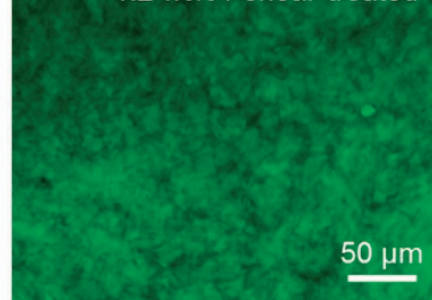

Figure 6 Confocal laser scanning microscopic (CLSM) analyses of GO alignment and curvatures. Large-sized GO dispersions were used, and the non-treated and shear-treated cells shown in Figure $3 \mathrm{~b}$ were used for the top and bottom row images, respectively. Images were obtained at a depth of $\sim 50 \mu \mathrm{m}$. (a) At $0.25 \mathrm{wt} \%$, the GO particles were clearly discernible. The magnified inset images ( $\mathrm{i}$-iii) on the right side show the sectional cuts of vertically aligned flat GOs and curved GOs and the horizontally aligned GOs, respectively. (b) Shear treatment induced significant horizontal alignment of the GO particles. Closely packed particles appear brighter than sparsely packed particles. (c, d) At $0.45 \mathrm{wt} \%$, individual particles were dimly discernible. The non-treated cell exhibited a vertically packed GO alignment, whereas shear treatment enhanced the horizontal alignment. (e, f) At 1.2 wt\%, individual particles were not discernible, but the brightness and uniformity provided information regarding the GO particle alignment and packing assembly.

concentration dispersions (Figure 6e and f), cloud-like agglomerated particles, rather than individual particles, were observed. With shear treatment, the CLSM image became uniform and brighter, indicating a uniform and close interlayer stacking assembly. The top-down shear force may have flattened not only the undulated GO alignment but also the curvature of the GO particles and thus improved the quality of the interlayer stacking assembly, which correlates with the shear-induced improvement in the spectral reflectance (Figure 3d).

Concentration-driven coloration is indicative of a swollen lamellar structure, which could extend either locally or over a long-range. The 

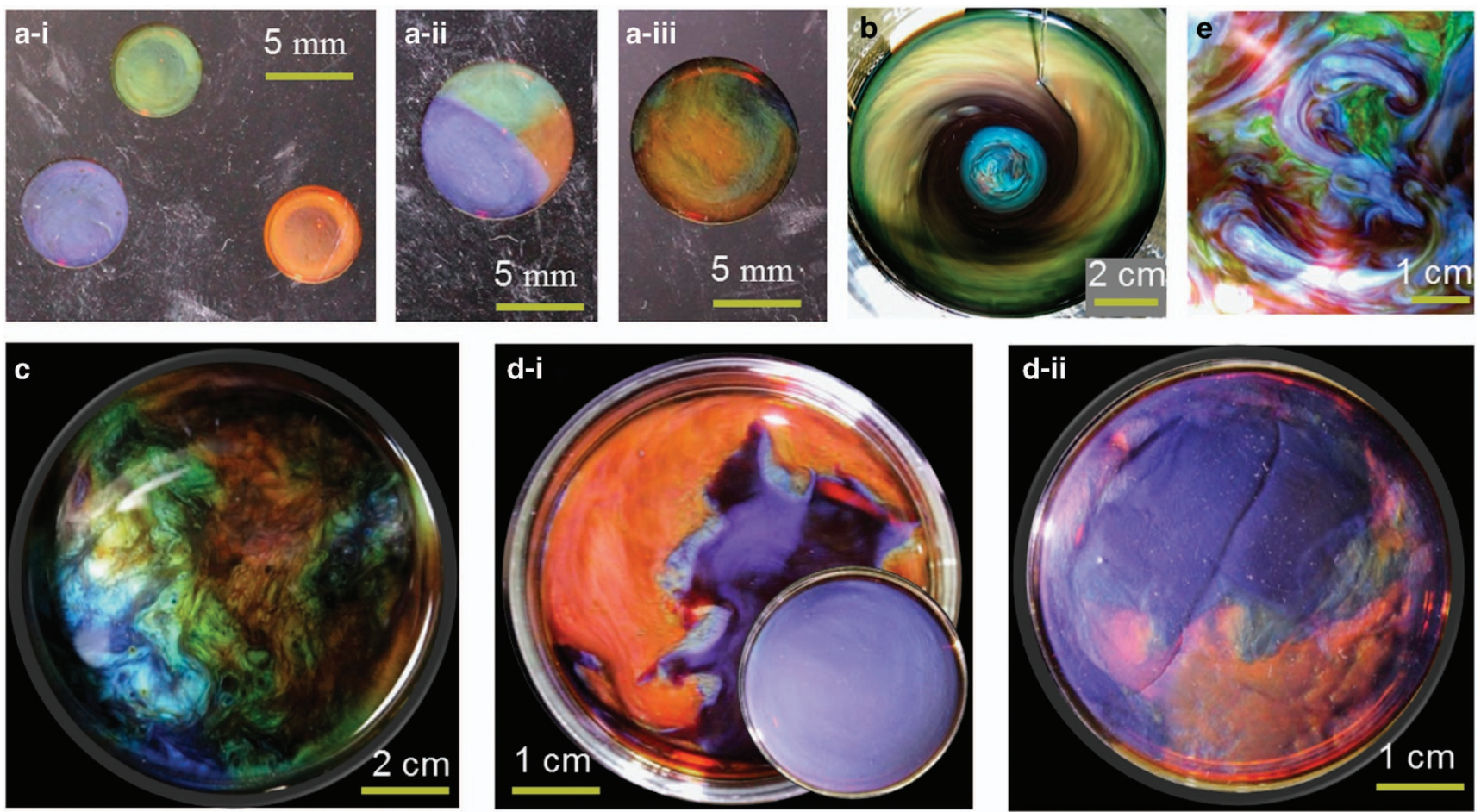

Figure 7 Various colored drawings using multiple GO dispersions. (a) GO droplets reflecting red, green and blue colors were dropped onto a hydrophobic substrate (a-i) and merged into one drop with clear border lines (a-ii). The colors were mixed when the substrate was shaken (a-iii). (b) Upon adding water into a stirred GO dispersion, the color changed continuously. (c) A GO dispersion with spatially varying GO concentration exhibited full color reflection, and the colorful drawing was sustained for 1 day. (d) Shallow GO dispersions, $\sim 1 \mathrm{~mm}$ thick, in a petri dish exhibited fairly uniform color in the top view (d-i) and the bottom view (d-ii). (e) By dropping another GO dispersion on top of the base dispersion, the colored character ' $\mathrm{S}$ ' was obtained. The topping color can either replace or mix with the base color.

CLSM image in Figure 6f shows mottled, bright grains within a dark background. The bright grains correspond to dense layer-to-layer stacking, whereas the dark region indicates the missing-layer GO assembly. As indicated in the inset image (iii) in Figure $6 \mathrm{a}$, the irregularly shaped GO particles do not fit with each other (unlike a jigsaw), and as a result, a layered structure with partially missing layers is unavoidable in vertical stacking. Therefore, it is difficult to observe a wide-range single layer in GO alignment, although local layer-to-layer stacking is excellent. A weak diffuse spot was reported in GO dispersions using a small angle X-ray scattering analysis. ${ }^{30,40}$ This broad, diffuse peak indicates that the GO liquid crystal has liquid-like positional local order in a globally nematic phase, which is not considered a lamellar mesophase with a long-range positional order. ${ }^{41,42}$ Hence, it is reasonable to consider the GO photonic crystal as a nematic phase rather than a lamellar mesophase. Figure 2 shows that the reflectance of the GO photonic crystal was $\sim 3 \%$, which is much lower than the usual photonic crystal structure $(\sim 31 \%$ for the CLC cell in Figure 3f). In fact, the low reflectivity in the GO photonic crystals implies defective local lamellar ordering instead of the long-range lamellar mesophase. Thus, unlike the excellent local layer-to-layer periodicity due to EDL packing assembly, in-layer assembly is rather poor and defective.

Colorful painting based on the local modulations of GO concentration and alignment

The mixing of GO dispersions with different GO concentrations displayed fascinating structural color properties. Three small GO droplets having different structural colors were dropped onto a hydrophobic substrate (Figure 7a-i) and were merged into one droplet by gently tilting the substrate (Figure 7a-ii). The merged droplet had three regions of different colors with clear boundary lines. Interestingly, the colors were not spontaneously mixed until the substrate was shaken to force the mixing (Figure 7a-iii). Upon adding water into a beaker containing a stirred high-concentration GO dispersion, the reflection color changed continuously and varied spatially, generating colorful reflections (Figure 7b). Moreover, we could draw a colorful painting by dropping varying amounts of water into the GO dispersion after stopping the magnetic stirring, as shown in the Earth-like painting in Figure 7c. The colorful picture was sustained for at least 1 day, indicating that spontaneous diffusion of GO particles occurs very slowly in water. Dropping water caused a complicated undulating flow-mark, generating dark and bright reflections. The dark reflection was due to the GO aligned perpendicular to the surface. Gentle shaking of a shallow GO dispersion $(\sim 1 \mathrm{~mm}$ depth) with a blue reflection in a petri dish induced a uniform color reflection (inset image in Figure $7 \mathrm{~d}-\mathrm{i}$ ). By adding a red GO dispersion on top of the thin blue GO dispersion, relativity uniform color domains were obtained, owing to the surface effect of the petri dish (Figure $7 \mathrm{~d}-\mathrm{i}$ ). Figure $7 \mathrm{~d}$-ii shows the bottom view of the petri dish, which shows almost the same color patterns as the top view in Figure $7 \mathrm{~d}$-i, albeit with a weaker red color owing to the presence of the blue GO dispersion layer underneath the red GO dispersion.

Dropping a GO dispersion with one color on top of another dispersion with a different color enabled us to draw a colored character (Figure 7e). In the case of a sufficiently thick topping layer, the reflection color was replaced by the topping layer. The color was mixed with that of the bottom layer, however, in the case of a thin 
topping layer. Modulations of the local GO density and alignment enabled us to demonstrate full-range color reflections, including chromatic and achromatic colors (see Supplementary Figure S6). The modulations of local GO density and alignment were stable and sustained, indicating weak diffusion of GO particles in the GO concentrations.

\section{DISCUSSION}

The achievement of a high-quality, multi-order, visible-range photonic bandgap in colloids of polydispersed, irregularly shaped and curved GO flakes is an unexpected result. The photonic bandgap does not arise from a higher-order hierarchical periodic structure but from periodic stacking of single-layered GO sheets, which is confirmed by the ratio of the interlayer spacing and the reciprocal volume fraction. The thickness of GO sheets is only ca. $1 \mathrm{~nm}$, but the uniform interlayer spacing is up to $500 \mathrm{~nm}$, sustained by electrostatic repulsion. Phase identification is critical in the study of liquid crystals, to understand the origin of the self-assembly, hierarchical structures, material properties and even potential applications. However, the unexpected photonic crystallinity complicates the phase identification of the GO dispersion; 1D photonic crystallinity is usually attributed to lamellar mesophasic ordering. ${ }^{43}$ Several additional results have been reported to support the lamellar mesophasic property of the GO dispersion, such as the periodic patterns with pitches of tens of micrometers in the freeze-dried sample ${ }^{38,44}$ and small angle $\mathrm{X}$-ray scattering peaks sharper than those in usual small-molecular nematic phases. ${ }^{30,38,45}$ However, freeze-dried samples usually lose the original GO assembly structure in a wet colloid and are restructured during freezing, ${ }^{36,37}$ small angle X-ray scattering peaks in colloidal nematic phases are commonly sharper than those of small-molecular nematic phases owing to weaker thermal fluctuation, and the types of patterns are commonly interpreted as characteristic patterns of the nematic phase rather than the lamellar phase in clay suspensions. ${ }^{41,42,46}$ Hence, these results do not directly confirm the lamellar mesophase of the GO dispersion. In lamellar phases such as lipid bilayer lamellar phases and the smectic phases of small molecules, a long-range single layer that is composed of many particles should first be defined. Then, the stacking assembly of the layers constitutes the bulk lamellar phase. GO dispersions have excellent layer-to-layer assembly but poor in-layer assembly, which makes it difficult to define each layer. Thus, GO dispersions are identified as belonging to a nematic phase rather than to a lamellar mesophase, despite their lamellar properties such as high-quality local layer-to-layer periodicity. Polydispersity, irregular shape and flexibility may disturb the formation of the lamellar mesophase. The excellent layer-to-layer packing assembly originates from a thick and uniform EDL on the GO surface.

Top-down manipulation of GO photonic crystallinity using surface and shear fields significantly contributes to the flexibility of 2D GO sheets. These external stimuli improve the GO alignment and smooth the curvature of GO particles, in turn leading to variations in the color and brightness of the reflected light. It is also interesting that the local variation of GO density can be sustained for a considerable period of time, provided that the GO is not stirred or shaken. This implies that the local osmotic pressure is weak or self-balancing across a wide range of concentrations. The results in this study may open a new avenue to using GO dispersions in reflective-type optical devices, although further study is required to resolve many issues, such as poor stability and low reflectance.

\section{CONFLICT OF INTEREST}

The authors declare no conflict of interest.

\section{ACKNOWLEDGEMENTS}

This work was supported by the Samsung Research Funding Center of Samsung Electronics under Project Number SRFC-MA1402-03.

Author contributions: J-KS planned and supervised the project. T-ZS and S-HH contributed equally to this work by performing most of the experiments. BL and T-ZS performed the CLSM analysis. All authors analyzed the data and participated in the writing of the manuscript.

1 Dimiev, A. M., Alemany, L. B. \& Tour, J. M. Graphene oxide. Origin of acidity, its instability in water, and a new dynamic structural model. ACS Nano 7, 576-588 (2013).

2 Loh, K. P., Bao, Q., Eda, G. \& Chhowalla, M. Graphene oxide as a chemically tunable platform for optical applications. Nat. Chem. 2, 1015-1024 (2010).

3 Shen, T.-Z., Hong, S.-H. \& Song, J.-K. Electro-optical switching of graphene oxide liquid crystals with an extremely large Kerr coefficient. Nat. Mater. 13, 394-399 (2014).

4 He, L., Ye, J., Shuai, M., Zhu, Z., Zhou, X., Wang, Y., Li, Y., Su, Z., Zhang, H., Chen, Y., Liu, Z., Cheng, Z. \& Bao, J. Graphene oxide liquid crystals for reflective displays without polarizing optics. Nanoscale 7, 1616-1622 (2015).

5 Li, P., Wong, M., Zhang, X., Yao, H., Ishige, R., Takahara, A., Miyamoto, M., Nishimura, R. \& Sue, H.-J. Tunable lyotropic photonic liquid crystal based on graphene oxide. ACS Photonics 1, 79-86 (2014).

6 Gabriel, J.-C. P., Camerel, F., Lemaire, B. J., Desvaux, H., Davidson, P. \& Batail, P. Swollen liquid-crystalline lamellar phase based on extended solid-like sheets. Nature 413, 504-508 (2001).

7 Haque, M. A., Kurokawa, T. \& Gong, J. P. Anisotropic hydrogel based on bilayers: color, strength, toughness, and fatigue resistance. Soft Matter 8, 8008 (2012).

8 Wong, M., Ishige, R., Hoshino, T., Hawkins, S., Li, P., Takahara, A. \& Sue, H.-J. Solution processable iridescent self-assembled nanoplatelets with finely tunable interlayer distances using charge- and sterically stabilizing oligomeric polyoxyalkyleneamine surfactants. Chem. Mater. 26, 1528-1537 (2014).

9 Mourad, M. C., Petukhov, A. V., Vroege, G. J. \& Lekkerkerker, H. N. Lyotropic hexagonal columnar liquid crystals of large colloidal gibbsite platelets. Langmuir 26, 14182-14187 (2010).

10 van der Kooij, F. M., Kassapidou, K. \& Lekkerkerker, H. N. W. Liquid crystal phase transitions in suspensions of polydisperse plate-like particles. Nature 406, 868-871 (2000).

11 Arsenault, A. C., Puzzo, D. P., Manners, I. \& Ozin, G. A. Photonic-crystal full-colour displays. Nat. Photonics 1, 468-472 (2007).

12 Rossi, L., Sacanna, S., Irvine, W. T. M., Chaikin, P. M., Pine, D. J. \& Philipse, A. P. Cubic crystals from cubic colloids. Soft Matter 7, 4139 (2011).

13 Takeoka, Y., Honda, M., Seki, T., Ishii, M. \& Nakamura, H. Structural colored liquid membrane without angle dependence. ACS Appl. Mater. Interfaces 1, 982-986 (2009).

14 Whitby, R. L. D., Gun'ko, V. M., Korobeinyk, A., Busquets, R., Cundy, A. B., László, K., Skubiszewska-Zięba, J., Leboda, R., Tombácz, E., Toth, I. Y., Kovacs, K. \& Mikhalovsky, S. V. Driving forces of conformational changes in single-layer graphene oxide. ACS Nano 6, 3967-3973 (2012).

15 Catheline, A., Ortolani, L., Morandi, V., Melle-Franco, M., Drummond, C., Zakri, C. \& Pénicaud, A. Solutions of fully exfoliated individual graphene flakes in low boiling point solvents. Soft Matter 8, 7882 (2012).

16 Hong, S. H., Shen, T. Z. \& Song, J. K. Manipulation of structural color reflection in graphene oxide dispersions using electric fields. Opt. Express 23, 18969-18974 (2015).

17 Hummers, W. S. \& Offema, R. E. Preparation of graphite oxide. J. Am. Chem. Soc. 80, 1339-1339 (1958).

18 Shen, T.-Z., Hong, S. H. \& Song, J.-K. Effect of centrifugal cleaning on the electro-optic response in the preparation of aqueous graphene-oxide dispersions. Carbon 80, 560-564 (2014).

19 Hong, S.-H., Shen, T.-Z. \& Song, J.-K. Electro-optical characteristics of aqueous graphene oxide dispersion depending on ion concentration. J. Phys. Chem. C 118, 26304-26312 (2014).

20 Shen, T.-Z., Hong, S.-H. \& Song, J.-K. Effect of centrifugal cleaning on the electro-optic response in the preparation of aqueous graphene-oxide dispersions. Carbon 80, 560-564 (2014).

21 Williams, G., Seger, B. \& Kamat, P. V. TiO2-Graphene nanocomposites. UV-assisted photocatalytic reduction of graphene oxide. ACS Nano 2, 1487-1491 (2008).

22 Ding, Y. H., Zhang, P., Zhuo, Q., Ren, H. M., Yang, Z. M. \& Jiang, Y. A green approach to the synthesis of reduced graphene oxide nanosheets under UV irradiation. Nanotechnology 22, 215601 (2011).

23 Hong, S.-H., Shen, T.-Z. \& Song, J.-K. Flow-induced ordering of particles and flow velocity profile transition in a tube flow of graphene oxide dispersions. Liq. Cryst. $\mathbf{4 2 ,}$ 261-269 (2014).

24 Kim, J.-K., Joo, S.-H. \& Song, J.-K. Complementarity between fluorescence and reflection in photoluminescent cholesteric liquid crystal devices. Opt. Express 21, 6243-6248 (2013)

25 Onsager, L. The effect of shape on the interaction of colloidal particles. Ann. N.Y. Acad. Sci. 51, 627-659 (1949). 
26 Bates, M. A. \& Frenkel, D. Nematic-isotropic transition in polydisperse systems of infinitely thin hard platelets. J. Chem. Phys. 110, 6553 (1999).

27 van der Kooij, F. M. \& Lekkerkerker, H. N. W. Formation of nematic liquid crystals in suspensions of hard colloidal platelets. J. Phys. Chem. B 102, 7829-7832 (1998).

28 Rowan, D. G. \& Hansen, J.-P. Salt-induced ordering in lamellar colloids. Langmuir 18, 2063-2068 (2002).

29 Kim, J. E., Han, T. H., Lee, S. H., Kim, J. Y., Ahn, C. W., Yun, J. M. \& Kim, S. O. Graphene oxide liquid crystals. Angew. Chem. Int. Ed. Engl. 50, 3043-3047 (2011).

$30 \mathrm{Xu}$, Z. \& Gao, C. Aqueous liquid crystals of graphene oxide. ACS Nano $\mathbf{5}$ 2908-2915 (2011).

31 Dan, B., Behabtu, N., Martinez, A., Evans, J. S., Kosynkin, D. V., Tour, J. M., Pasquali, M. \& Smalyukh, I. I. Liquid crystals of aqueous, giant graphene oxide flakes. Soft Matter 7, 11154 (2011).

32 Luo, Y., Braggin, G. A., Olson, G. T., Stevenson, A. R., Ruan, W. L. \& Zhang, S. Nematic order drives macroscopic patterns of graphene oxide in drying drops. Langmuir 30, 14631-14637 (2014).

33 Forster, J. D., Noh, H., Liew, S. F., Saranathan, V., Schreck, C. F., Yang, L., Park, J. G., Prum, R. O., Mochrie, S. G., O'Hern, C. S., Cao, H. \& Dufresne, E. R. Biomimetic isotropic nanostructures for structural coloration. Adv. Mater. 22, 2939-2944 (2010).

34 Yethiraj, A. \& van Blaaderen, A. A colloidal model system with an interaction tunable from hard sphere to soft and dipolar. Nature 421, 513-517 (2003).

35 Hong, S.-H., Shen, T.-Z. \& Song, J.-K. Water front recession and the formation of various types of wrinkles in dried graphene oxide droplets. Carbon 105, 297-304 (2016)

36 Qiu, L., Liu, J. Z., Chang, S. L., Wu, Y. \& Li, D. Biomimetic superelastic graphenebased cellular monoliths. Nat. Commun. 3, 1241 (2012).

37 Ham, H., Khai, T. V., Park, N. H., So, D. S., Lee, J. W., Na, H. G., Kwon, Y. J., Cho, H. Y. \& Kim, H. W. Freeze-drying-induced changes in the properties of graphene oxides. Nanotechnology 25, 235601 (2014).

$38 \mathrm{Xu}, \mathrm{Z}$. \& Gao, C. Graphene chiral liquid crystals and macroscopic assembled fibres. Nat. Commun. 2, 571 (2011).

39 Tong, L., Qi, W., Wang, M., Huang, R., Su, R. \& He, Z. Long-range ordered graphite oxide liquid crystals. Chem. Commun. (Camb) 50, 7776-7779 (2014).
40 Zakri, C., Blanc, C., Grelet, E., Zamora-Ledezma, C., Puech, N., Anglaret, E. \& Poulin, P. Liquid crystals of carbon nanotubes and graphene. Philos. Trans. A Math. Phys. Eng. Sci. 371, 20120499 (2013).

41 Paineau, E., Philippe, A. M., Antonova, K., Bihannic, I., Davidson, P., Dozov, I., Gabriel, J. C. P., Impéror-Clerc, M., Levitz, P., Meneau, F. \& Michot, L. J. Liquid-crystalline properties of aqueous suspensions of natural clay nanosheets. Liq. Cryst. Rev. 1, 110-126 (2013).

42 Paineau, E., Michot, L. J., Bihannic, I. \& Baravian, C. Aqueous suspensions of natural swelling clay minerals. 1. Structure and electrostatic interactions. Langmuir 27, 5562-5573 (2011).

43 Li, Z., Liu, Z., Sun, H. \& Gao, C. Superstructured assembly of nanocarbons: fullerenes, nanotubes, and graphene. Chem. Rev. 115, 7046-7117 (2015).

$44 \mathrm{Xu}$, Z. \& Gao, C. Graphene in macroscopic order: liquid crystals and wet-spun fibers. Acc. Chem. Res. 47, 1267-1276 (2014).

45 Liu, Z., Xu, Z., Hu, X. \& Gao, C. Lyotropic liquid crystal of polyacrylonitrile-grafted graphene oxide and its assembled continuous strong nacre-mimetic fibers. Macromolecules 46, 6931-6941 (2013).

46 Lekkerkerker, H. N. \& Vroege, G. J. Liquid crystal phase transitions in suspensions of mineral colloids: new life from old roots. Philos. Trans. A Math. Phys. Eng. Sci. 371, 20120263 (2013).

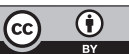

This work is licensed under a Creative Commons Attribution 4.0 International License. The images or other third party material in this article are included in the article's Creative Commons license, unless indicated otherwise in the credit line; if the material is not included under the Creative Commons license, users will need to obtain permission from the license holder to reproduce the material. To view a copy of this license, visit http:// creativecommons.org/licenses/by/4.0/

(C) The Author(s) 2016

Supplementary Information accompanies the paper on the NPG Asia Materials website (http://www.nature.com/am) 